\title{
Le document authentique, un outil médiateur des interactions en classe de FLE
}

\author{
Karen Kênnia Couto Silva ${ }^{1}$
}

\begin{abstract}
Résumé : Le principal objectif de cet article est de circonscrire la place des documents authentiques en classe de Français Langue Étrangère (FLE) et de répondre à la question centrale : les interactions en classe de langue sont-elles favorisées par l'utilisation des documents authentiques ? Si oui, comment ? Dans la première partie de ce travail, nous tenterons de clarifier la notion des documents authentiques et ses différentes perspectives (l'origine de ce concept, la variété des définitions, les atouts et les limites, le dégré d'authenticité et l'insertion dans l'apprentissage d'une langue étrangère). Dans la seconde partie de ce travail, nous analyserons les interactions en classe de FLE engendrées par l'utilisation des documents authentiques. Pour ce faire, nous nous appuierons sur des enregistrements vidéo et sur des questionnaires soumis aux professeurs de FLE. Les résultats de notre étude nous indiquent que l'utilisation des documents authentiques permet l'exploitation de différents thèmes en cours, favorise le travail en parallèle de la langue et de la culture et favorise une communication plus réelle et une interaction décontractée. Lorsque les élèves manipulent de nombreux documents, ils se sentent impliqués par ces supports, travaillent de façon autonome leur processus de l'acquisition de la langue et de la culture et agissent comme de vrais acteurs. Enfim, nous partageons de l'opinion de plusieurs chercheurs selon laquelle les documents authentiques sont des outils didactiques qui aident à l'apprentissage d'une langue étrangère.
\end{abstract}

Mots-clefs : Document authentique ; Interaction ; Stratégie d'enseignement ; Compétence Orale.

\section{O documento autêntico, uma ferramenta mediadora das interações em aula de FLE}

Resumo: O principal propósito deste estudo é investigar o papel dos documentos autênticos em sala de Francês Língua Estrangeira (FLE) a fim de responder a questão central: as interações em sala de línguas são favorecidas pela utilização de documentos autênticos? Se sim, de qual forma? Na primeira parte deste estudo pretende-se clarear a noção de documentos autênticos em diferentes perspectivas (a origem do conceito, as inúmeras definições, as vantagens e as desvantagens, o grau de autenticidade e a inserção no ensino-aprendizagem de uma língua estrangeira). Na segunda parte deste trabalho, a partir de gravações de aulas e questionários submetidos a professores analisam-se as interações em sala de FLE emergidas a partir da utilização de documentos autênticos. Os resultados encontrados indicam fortemente que a utilização de documentos autênticos possibilita a abordagem de diferentes temas, favorecem o trabalho concomitante com a língua e a civilização e permitem uma comunicação real e uma interação descontraída. Ao manusearem um documento autêntico, os alunos se sentem mais motivados a participarem da aula, são mais autônomos em seu processo de aquisição da língua e da cultura e agem como verdadeiros atores. Assim, o presente estudo corrobora o esforço de outros pesquisadores ao sugerir que os documentos autênticos são ferramentas didáticas de estimado valor no ensino-aprendizagem de língua estrangeira.

Palavras-chave: Documento autêntico ; Interação ; Estratégia de ensino ; Competência oral.

1 Karen COUTO SILVA a été étudiante en Master recherche intitulé "Sciences du langage - Specialité Français Langue Étrangère de l'Université Stendhal - Grenoble 3. Année universitaire: 2012/2013. Cet article, réalisé sous la direction de Claudine Moïse, porte sur l'interaction en classe de FLE, l'un des sujets abordés dans le cadre du Master FLE.

Contact : karenbhz@yahoo.com.br 


\section{Introduction}

L'enseignement et l'apprentissage du Français Langue Etrangère ont été, à partir des années 70, un terrain d'application productif pour les théories sur le fonctionnement des langues. Depuis cette date jusqu'à nos jours, l'acquisition de la langue est liée aux objectifs communicatifs, c'est-à-dire que « la langue sert à transmettre des messages, donc à exprimer des intentions de communication à des partenaires avec lesquels on se trouve en interaction...» (COURTILLON, 2003, p. 6).

La communication humaine se réalise en grande partie à travers le langage verbal (textes oraux et écrits) et le langage non-verbal (geste, mimes, émotions). En nous appuyant sur la notion de texte adoptée par le Cadre Européen Commun de Référence, «toute séquence discursive orale ou écrite que les usages reçoivent, produisent ou échangent »(CECR, 2001, p.75), nous porterons notre recherche sur l'étude des textes écrits dans le cadre de l'enseignement du Français Langue Étrangère (FLE).

Le présent article porte sur l'analyse des interactions en classe de FLE produites par l'utilisation des documents authentiques (DAs). Comme l'un des objectifs essentiels du FLE est de développer chez les apprenants la capacité de s'exprimer dans cette langue, nous essaierons d'apporter quelques éléments qui favorisent l'acquisition de la compétence orale.

La première partie de ce travail, sera d'ordre théorique. Elle débutera par un cadre conceptuel dans lequel nous essaierons de clarifier les notions suivantes : les DAs et l'interaction en classe de FLE. Dans la seconde partie de cette étude, d'ordre pratique, nous ferons l'analyse des interactions en classe de FLE et nous nous appuierons également sur des questionnaires soumis aux enseignants et aux apprenants à propos de l'objet d'enseignement document authentique.

Voici les différentes interrogations auxquelles nous tenterons de répondre : quel est le degré d'authenticité des documents « authentiques »? Les enseignants ont-ils recours aux textes authentiques dans leur pratique ? Comment les utilisent-ils ? Comment cerner l'agir enseignant en relation avec les conduites des étudiants et avec les objets mobilisés (les DAs)? Les documents authentiques constituent-ils des matériels privilégiés pour l'enseignement de Français Langue Étrangère? 
Notre but est de montrer la place tenue par les DAs pour l'enseignement du FLE et de répondre à la question centrale : les interactions en classe sont-elles favorisées par l'utilisation des DAs ? Si oui, comment?

\section{L'authenticité dans l'apprentissage du FLE}

En didactique de langues, un document correspond, la plupart du temps, à un support sur lequel les enseignants s'appuient pour préparer leur cours et les didacticiens utilisent dans leurs manuels. L'objectif de cette partie est d'explorer la notion de DAs : l'apparition de ce concept, tout en passant par la définition du DA, ses atouts, ses limites, jusqu'à en discuter le choix et son insertion dans l'enseignement du FLE.

\section{Origine et définition}

Les didacticiens Cuq et Gruca affirment que les DAs émergent dans une période de transition entre la méthodologie SGAV et la méthodologie communicative. Au cours des années 70, il y avait « la nécessité d'unir étroitement l'enseignement de la langue à celui de la civilisation et de mettre en contact l'élève avec la langue réelle ». (Cuq \& Gruca, 2005, p.429)

Nous apporterons ici des définitions du mot authentique, de manière à élargir notre étude. Le but est de croiser les définitions déjà existantes en vue d'une précision réflexive. Nous commencerons avec Jean-Pierre Cuq qui, dans sa définition, associe les DAs aux genres du discours « La caractérisation d' «authentique», [...], est généralement associée à " document » et s'applique à tout message élaboré par des francophones pour des francophones à des fins de communication réelle »(CUQ, 2003, p.29).

Mais jusqu'où pouvons-nous dire que les documents sont authentiques ? Henri Besse affirme que le DA est désauthentifié à partir du moment où il est détaché de son contexte. En ce sens, « l'authentique n'existe pas », c'est-à-dire qu'un texte est toujours fabriqué par un auteur et l'authenticité consiste à utiliser un texte tel qu'il a été produit. Geneviève Zarate doute aussi de l'authenticité des DAs «Le DA est souvent l'objet de trucages ». (ZARATE, 1986, p.76). Elle affirme que le DA perd son authenticité quand il est modifié pédagogiquement par un enseignant visant l'enseignement de ses étudiants. 
En adoptant le regard pratique de Bérard qui s'attache à une fonction pratique des DAs dans un cours de langue, nous formulerons ici notre définition : les DAs sont des textes que l'on trouve dans la société et qui peuvent être didactisés. Lorsqu'ils sont modifiés pour une méthodologie cohérente, ils perdent leur contexte de production mais gagnent des caractéristiques pédagogiques. Quand ils sont utilisés de façon consciente par l'enseignant, ce sont de vrais outils qui amènent l'apprenant à approfondir le fonctionnement de la langue.

\section{La variété des documents authentiques}

Les DAs font partie d'un ensemble de textes présents dans les situations de communication. Ils fournissent une énorme variété de textes et de discours. Pour cette raison, ils sont appelés « documents sociaux ». Cuq et Gruca les classent en :

[...] documents de la vie quotidienne (plan d'une ville, horaires de train, etc.) documents administratifs (fiche d'inscription, formulaires, etc.)

[...] médiatiques écrits, sonores, télévisés (articles, bulletins météorologiques, etc.) oraux (interviews, chansons,etc.) ou iconographiques (photos, tableaux, etc.) (CUQ ; GRUCA, 2003, p.432)

Comme on vient de le voir, les documents sont multiples et ils peuvent être utiles dans des activités de compréhension et d'expression orale et écrite ainsi que des activités qui traitent des contenus grammaticaux et lexicaux. Dans la didactique des langues, il est primordial de rattacher le choix de ces documents à une méthodologie cohérente et précise : «il n’a de sens que pris dans un projet méthodologique qui lui donne une fonction et une place» (COSTE, 1970, p. 90).

\section{Atouts et limites des documents authentiques}

Dans cette partie, nous allons montrer les avantages et les inconvénients de l'utilisation des DAs. Nous nous poserons les questions suivantes : pourquoi les DAs sont-ils privilégiés pour l'enseignement des langues étrangères? Quels sont les facteurs qui empêchent l'utilisation de ces documents? Nous énumérerons ces aspects dans ce tableau ci-dessous (Tab.1):

\begin{tabular}{|l|l|}
\hline \multicolumn{1}{|c|}{ Atouts } & \multicolumn{1}{c|}{ Limites } \\
\hline $\begin{array}{l}\text { Permet une initiation à l'approche d'une autre } \\
\text { culture }\end{array}$ & Les difficultés de l'accès au DA à l'étranger \\
\hline C'est un outil déclencheur de motivation & $\begin{array}{l}\text { La question des droits d'auteur freinant } \\
\text { l'utilisation des DAs }\end{array}$ \\
\hline Permet l'autonomie des apprenants & Le vieillissement des Das \\
\hline $\begin{array}{l}\text { Permet le croisement de la langue et de la } \\
\text { culture }\end{array}$ & L'insertion du DA dans l'ensemble du cours \\
\hline
\end{tabular}




\begin{tabular}{ll|l|}
\hline $\begin{array}{l}\text { C'est un moyen de communication } \\
\text { authentique en cours }\end{array}$ & $\begin{array}{l}\text { La complexité discursive et linguistique du } \\
\text { DA }\end{array}$ \\
\hline
\end{tabular}

\section{Documents authentiques et enseignement du FLE}

Cette recherche conçoit l'enseignant comme un formateur responsable pour simplifier un contenu, pour chercher des méthodologies plus efficaces et pour capter l'attention de l'étudiant. Il doit utiliser ses savoirs lorsqu'il choisit les DAs, et ce choix ne doit pas être aléatoire: «le choix même des documents mal adaptés aux possibilités extralinguistiques des apprenants risque de les cantonner dans un rôle de lecteurs assistés »(BESSE, 1980.p. 81).

Autrement dit, le choix fait par l'enseignant doit prendre en compte la situation d'apprentissage, le niveau des apprenants ainsi que leurs besoins.

La « transformation » des DAs peut être nécessaire, lorsque l'enseignant prépare le cours, soit pour l'adapter au niveau enseigné, soit à cause de la longueur du document choisi. Et s'il est nécessaire de modifier le document, il faut le faire sinon on risque de négliger l'utilisation de ces outils et les rendre "impossibles » aux apprenants. Il en résulte que les DAs peuvent perdre leur authenticité originale. Pour ne pas en arriver là, l'enseignant doit adopter une démarche pédagogique qui respecte « les éléments de la situation dans laquelle s'insère son énoncé » (CUQ ; GRUCA, 2005, p.434).

Nous avons constaté que le choix du DA doit être cohérent avec ce qu'on veut enseigner et viser les besoins des élèves; il faut réfléchir à « comment » utiliser ces outils dans la classe de FLE. Cuq met en évidence le fait qu' « il faut éviter à tout prix le piège de l'utilisation artificielle de l'authentique» (CUQ ; GRUCA, 2005, p. 433).

Le plus important lorsque l'enseignant exploite les documents est de traiter le contenu du message, en articulant l'enseignement entre la langue et la culture. Ensuite, il peut tirer profit de ces documents pour développer la compréhension orale et écrite, travailler les éléments de situation de communication et enseigner les actes des paroles et les énoncés. En faisant cela, l'enseignement suit les orientations de l'approche communicative.

\section{Le document authentique, un outil médiateur des interactions en classe}


Le sociologue Erving Goffman a été l'un de premiers chercheurs à s'intéresser à l'interaction entre individus et à la manière dont la conversation progresse. Dans une volonté de circonscrire les échanges entre locuteurs, Goffman a recours à une métaphore « la vie sociale est un théâtre, et l'interaction une représentation »(GOFFMAN, 1973, p.47). Il part du principe que chaque individu a un rôle et doit chercher des informations pour s'engager et pour situer l'autre dans l'interaction.

L'interaction a été également l'objet d'étude de Kerbrat-Orecchioni. Celle-ci formule un postulat fondamental de l'approche interactionnelle " tout discours est une construction collective, ou une réalisation interactive (KERBRAT-ORECCHIONI, 1995, p.13). Ainsi, en s'appuyant sur le postulat, la chercheuse met en place la notion d'interaction :

Tout au long du déroulement d'un échange communicatif quelconque, les différents participants, que l'on dira donc des «interactants », exercent les uns sur les autres un réseau d' "influences mutuelles »-parler c'est échanger, et c'est changer en échangeant. (KERBRAT-ORECCHIONI, 1995, p. 17).

Vion définit l'interaction comme «...toute action conjointe, conflictuelle et/ou coopérative, mettant en présence deux ou plus de deux acteurs. (VION, 1942, p.17) Pour Cicurel (2005, p.4), l'analyse des interactions compte sur les acteurs bien réels et leur discours. Elle met en évidence que les situations socioculturelles jouent un rôle très important dans l'interaction : elles déterminent des comportements langagiers en rapport avec les contextes. Cicurel essaie de circonscrire l'apport de l'analyse interactionnelle :

L'entrée interactionniste porte davantage sur la relation entre des sujets sociaux que sur l'objet du discours qui, s'il est pris en compte, n'est que l'un des « ingrédients » de la description. Ce sont les manières de donner la parole, de faire parler les autres qui font l'objet d'observation (CICUREL, 2005, p.2).

À partir de l'ensemble des propositions explicitées ci-dessus à propos de la notion d'interaction, nous adoptons celle de Vion: «L'interaction est un lieu où se construisent le social, les représentations et les rapports de places. Ce point de vue est conforme à l'idée selon laquelle tout comportement modifie les données de l'interaction »(VION, 1992, p. 75).

\section{Composantes de l'interaction}

L'interaction est construite par un cadre communicatif (situation, site, but, participants, type d'interaction, etc.). Nous allons décrire ici chaque composante de l'interaction. Ensuite, nous décrirons ce qui se passe l'intérieur de l'activité dialogale : la succession et le mécanisme 
d'alternance de tour de parole. Pour cela, nous allons nous appuyer sur les théories de Vion (1992) et de Kerbrat-Orecchioni (1990).

Tout d'abord, l'analyse de la situation. Selon Vion (1992, p.79), une situation donnée est une situation construite par les participants et la situation générale. En ce sens, pour analyser une situation donnée, il faut nécessairement tenir compte du contexte dans lequel elle est émergée. Pour Kerbrat-Orecchioni, le site est le cadre spatio-temporel. Le cadre spatial peut être analysé par l'angle physique ou par l'angle social. Vion élargit la notion de site et il propose l'étude d'un espace interactif : Celui-ci comprend les sources énonciatives distinctes, le jeu de représentations et la coexistence de plusieurs rapports de places.

Le but peut être construit dans l'interaction (negocié entre les participants) et à l'extérieur de l'échange (préetabli préalablement). Avant d'initier l'interaction, il est souhaitable de se demander : de quoi parle-t-on? Dans quel but ? Ces questions, de nature simple, sont des clés pour la réussite de l'échange. Nous ne pouvons pas oublier que l'interaction ne peut être réalisée qu'en vue d'un but : interaction à fonction d'«obtenir l'information », à fonction d' " argumenter », à fin de « service », etc.

Les participants sont analysés dans leurs aspects objectifs (nombre et nature) par Kerbrat-Orecchioni. Celle-ci défend que ces aspects ne doivent pas être négligés, car ils déterminent le type d'interaction. Vion dépasse l'analyse des participants dans leurs aspects de nombre et de nature. Il souligne le caractère actif des participants « les sujets produisent du social dès lors qu'ils participent activement à la définition de la situation dans laquelles ils sont engagés, qu'ils sont les acteurs de l'actualisation des images et des représentations sans lesquelles il ne saurait y avoir de communication »(VION, 1992, p.64).

L'axe de la formalité est également objet d'étude dans les interactions. Selon KerbratOrecchioni (1990, p.71), les interactions peuvent être plus ou moins formelles. Vion illustre un type d'interaction informelle : " la conversation implique-t-elle un très petit nombre de participants, un cadre interactif symétrique, des règles implicites » et il met en place des exemples d'interaction formelle : « le débat ou la réunion nécessitent une gestion explicite et formalisée de la parole »(VION, 1992, p.128).

Le système de droits et de devoirs auxquels les participants sont soumis a été décrit par Kerbrat-Orecchioni (1990, p.73). Celle-ci affirme que les locuteurs doivent gérer entre eux la prise de parole. Pour cela, ils peuvent se servir de quelques propriétés : contre-balancement du 
discours de participants, l'individualisation de la parole et la prise de parole en continu entre les participants. Le changement de tour de parole peut être réalisé par des signaux de nature verbale (la complétude de l'énoncé), des signaux de nature phonétique (l'intonation marquée), des signaux de nature mimo-gestuelle (le regard soutenu).

Il faut mettre en évidence que, dans le système des tours de parole, les ratés sont fréquents. Ils se produisent dans le moment de l'alternance (silence prolongé entre deux tours, interruption, chevauchement de parole, intrusion) ou dans la succession de la parole (quand aucun candidat ne se présente ou plusieurs candidats se présentent à la fois).

On ne peut pas oublier que la conversation compte sur des règles de la grammaire conversationnelle - syntaxiques, sémantiques et pragmatiques. À titre d'exemple, nous citons les règles suivantes : la cohérence entre interventions de chaque participant et la cohérence interne à une même intervention, la mise en place des mécanismes de réprise et de correction. En dernier, il faut mettre en exergue les unités de l'interaction dialogales (interaction, séquence, échange) et monologales (intervention et actes de langage). Nous nous intéresserons ici à l'unité de l'interaction dialogale : l'interaction.

Pour Vion (1992, p.129), l'interaction peut être appréhendée en termes de rencontre et elle se déroule entre des individus déterminés. Kerbrat-Orecchioni (1990, p.215) propose des critères définitoires à cette unité : marquée par le temps et par le lieu, l'interaction est delimitée par la rencontre des participants et elle est déterminée par le critère thématique. Nous considérons que la classe de langue est un lieu d'interaction, c'est-à-dire « un lieu socialisé où s'établit un échange actif entre des partenaires ayant leur place dans l'interaction »(CICUREL, 2002, p.3).

\section{Le document authentique favorise-t-il les interactions en classe?}

Les situations didactiques ne sont pas naturelles ; l'enseignant et les apprenants sont soumis à des règles et à des normes. Nous formulons la question suivante : Pourquoi le DA devient-il un outil fondamental à ce type de situation ? Nous faisons ici plusieurs hypothèses : en premier, le contrat didactique, gouverné par des règles et des normes, est de plus en plus contraint. L'utilisation du DA en classe de langue signifie une ouverture à une situation de communication « authentique » et « réelle ».

On part du principe que le DA est un outil didactique qui permet l'étude de la langue et de la civilisation d'un pays. De plus, il est un objet d'enseignement qui favorise l'apprentissage des aspects linguistiques (grammaire, types de discours et registre de langue) et des aspects 
culturels et interculturels (en tant que fenêtre ouverte vers la France et sa civilisation, ainsi que les cultures francophones et d'autres cultures représentées par chaque étudiant de la classe).

Nous soulignons la valeur empirique DA. Sans lui, la relation entre les apprenants et l'enseignant devient moins chaleureuse et plus conflictuelle et l'interaction devient superficielle et non soutenante. Nous mettons ainsi le DA au centre de l'interaction. C'est lui qui va pousser l'interaction entre les partenaires, qui va donner le sens à la rencontre interculturelle, qui sert d'appui à l'explication d'un fait linguistique, etc.

Nous défendrons l'idée selon laquelle le DA régule les interactions en classe, c'est-àdire qu'il n'est pas seulement un moyen de savoir-dire de l'enseignant, il accorde un droit à parole de l'étudiant. Ainsi, avec son usage en classe de langue, les places des individus sont moins marquées et les positions des partenaires sont modulables. Enfin, le cours devient plus dynamique et l'enseignement plus horizontal.

Le document authentique est parfaitement inséré dans le programme du cours (très strict) et il rend possible une flexibilité communicative en classe de langue (très ouverte), dans le cas où l'enseignant décide de ne pas suivre le contenu programmé et laisse les apprenants parler « librement».

L’usage de DA encourage le schéma ternaire de l'interaction (question, réponse, action). Dans ce format ternaire, de nombreux actes de langage sont utilisés soit par l'enseignant soit par l'apprenant dans le but de leur faire comprendre. De cette manière là, des questions (directes/indirectes) sont formulées, des réponses (explicites/ implicites) sont mises en place, des reformulations sont crées et des réprises de l'enoncé précédent sont refaites.

\section{Méthodologie}

Notre recherche porte sur l'utilisation des DAs en classe de langue pour susciter la parole et l'interaction entre les apprenants. Pour cela, une observation participative a été menée dans des classes de français enseigné comme langue étrangère à l'Alliance Française de Belo

Horizonte $(\mathrm{BH})$ afin de recueillir des corpus de séquences de cours dans lesquelles les apprenants analysent des DAs.

L’Alliance Française de BH a été fondée en 1944. Elle difuse la langue française et les cultures francophones. Avec plus de 1200 étudiants brésiliens accueillis chaque année, elle est la première grande école d'enseignement de français de Belo Horizonte. Elle propose des cours de 
français général, des ateliers de français oral ou écrit, culturels et professionnels, des cours en entreprise et des formules personnalisées.

Nous avons observé les classes ayant le niveau A2. Le choix de ce niveau se justifie dans la mesure où $A 2$ est un niveau intermédiaire du niveau élémentaire, c'est-à-dire A2 est la suite du niveau «découverte» et cela nous semble indispensable d'avoir un regard critique car ce niveau est la base de l'apprentissage d'une langue. Autrement dit, à partir du niveau A2, l'apprenant est capable de s'exprimer d'une façon minimale dans la langue cible.

La classe A.2.1 est composée de huit apprenants et la classe A.2.2 est composée de 9 étudiants. La première classe est majoritairement jeune (6 étudiants entre 19 et 27 ans et 2 étudiants de 49 et 61 ans); la seconde classe est très diversifiée, nous avons 4 jeunes étudiants (entre 17 et 25 ans) et 5 étudiants plus âgés (entre 57 et 61 ans).

Dans notre recherche précédente, nous avons classés les documents présents en manuels de FLE en quatre catégories : DAs, documents semi-authentiques, documents fabriqués et autres documents. Nous avons proposé des critères définitoires à chaque type de documents (Tab.2) :

\section{Tableau 2 : documents authentiques, semi-authentiques, fabriqués et autres}

- Documents authentiques : les documents accompagnés de leur source, qui ont un « but communicatif »; les documents sans aucune transformation, adaptation, modification.

- Documents semi-authentiques : les documents authentiques accompagnés de source et des mots d'après ou extrait de.

- Documents fabriqués : les documents produits pour les auteurs eux-mêmes, à la finalité pédagogique bien précise et spécifique.

- Autres documents : Les documents qui sont situés à la limite des DAs et des documents fabriqués, ils peuvent être diffusés dans la société (les DAs) et ils peuvent être construits/utilisés dans un but pédagogique en préservant leurs caractéristiques, forme et sens (les documents fabriqués), c'est-à-dire qu'on ne peut pas distinguer leur origine.

Tableau réitéré de la page 27 du mémoire recherche de Karen COUTO SILVA, présenté dans le cadre du Master 1 - Sciences du Language, Spécialité Français Langue Étrangère, Université Stendhal - Grenoble 3. Année Universitaire 2011-2012.

Dans cet étude, nous nous centrerons sur les DAs et semi-authentiques. Notre but est de montrer leur rôle pour le déroulement de l'interaction en classe de langue et de répondre aux questions suivantes : comment faire parler les apprenants ? Comment susciter des interactions en classe de langue? 
Comme l'Alliance Française de BH adopte le manuel Écho, nous avons décidé d'analyser les interactions en classe engendrées par les DAs présents dans ce manuel. Nous avons enregistré les interactions en classe qui ont recours aux DAs suivants (Tab.3) :

\begin{tabular}{|c|c|c|c|c|c|}
\hline \multicolumn{6}{|c|}{ Tableau 3} \\
\hline Cours & Niveau & $\begin{array}{c}\text { Écho/A2 } \\
\text { Leçon }\end{array}$ & Titre du Document & $\begin{array}{c}\text { Type du } \\
\text { document }\end{array}$ & Source \\
\hline $1^{\mathrm{er}}$ & A.1.1 & $\begin{array}{l}\text { Leçon } 1 \\
\text { p. } 16\end{array}$ & $\begin{array}{c}\text { Quelle école pour } \\
\text { demain? }\end{array}$ & Article & Le Quotidien, 18/10/2007 \\
\hline $2^{\text {ème }}$ & A. 2.2 & $\begin{array}{l}\text { Leçon } 7 \\
\text { p. } 66-67\end{array}$ & Les nouvelles & Article & $\begin{array}{c}\text { Actu-wanadoo.fr, } \\
17 / 09 / 2005 \\
\end{array}$ \\
\hline 3 ème & A. 2.2 & $\begin{array}{l}\text { Leçon } 7 \\
\text { p. } 72-73\end{array}$ & $\begin{array}{l}\text { Naissance d'un chef } \\
\text { d'oeuvre }\end{array}$ & Histoire & $\begin{array}{l}\text { Au bonheur des mots, } \\
\text { Robert Laffont (1989) }\end{array}$ \\
\hline 4ème & A.2.2 & $\begin{array}{r}\text { Leçon } 8 \\
\text { p.80-81 } \\
\end{array}$ & $\begin{array}{l}\text { Un regard d'un } \\
\text { américain }\end{array}$ & Article & $\begin{array}{c}\text { Ted Stranger, Sacrés } \\
\text { Français, (2003) }\end{array}$ \\
\hline 5 ème & A. 2.2 & $\begin{array}{l}\text { Leçon } 9 \\
\text { p. } 96\end{array}$ & $\begin{array}{c}\text { Environ } 35.000 \\
\text { concurrents au } \\
\text { Marathon de Paris }\end{array}$ & Article & $\begin{array}{c}\text { AFP (Agence France } \\
\text { Presse)18/04/2007 }\end{array}$ \\
\hline
\end{tabular}

Nous allons mener cette analyse de la façon suivante. Nous analyserons le $1^{\text {er }}$ et le $5^{\text {ème }}$ cours. Pour cela, nous allons suivre l'organisation des parties théoriques: une session destinée à l'analyse des DAs présents dans le manuel Écho et une partie centrée sur les interactions produites par ces documents.

D’abord, nous ferons une présentation du document et une analyse de la pertinence des activités concernant le document analysé. Ensuite, nous choisirons quelques parties des corpus recueillis au long de la recherche portant sur le DA et nous ferons une analyse globale des échanges. Dans cette analyse, nous montrerons en quoi l'interaction va, ou pas, dans le sens d'une progression conversationnelle. Voici les conventions de transcription adoptées dans notre recherche (Tab.4):

\section{\begin{tabular}{|rll}
\hline & + & micro-pause \\
2 & $(2 s)$ & pauses en secondes \\
3 & $/ \backslash$ & intonation montante/ descendantel
\end{tabular}}




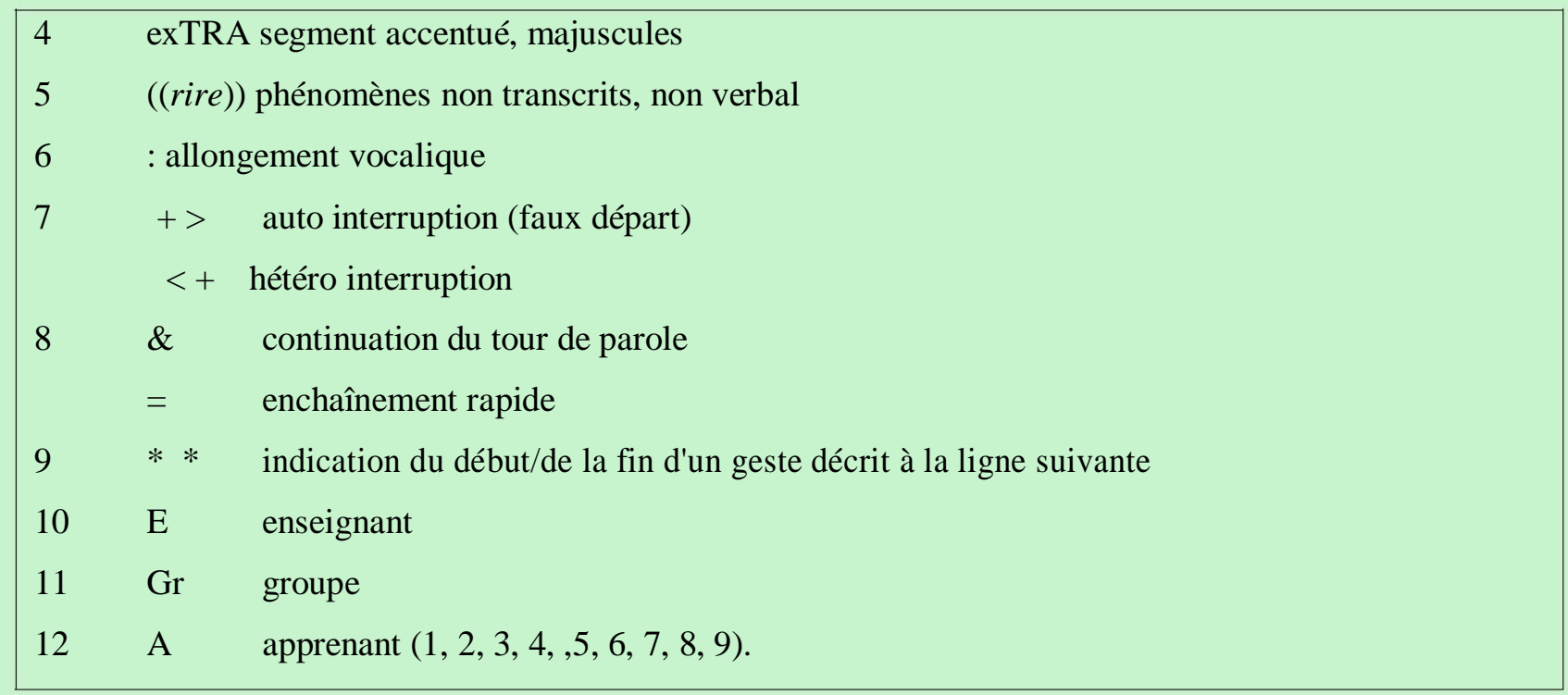

$1^{\text {er }}$ cours - Document : Article Quelle école pour demain?

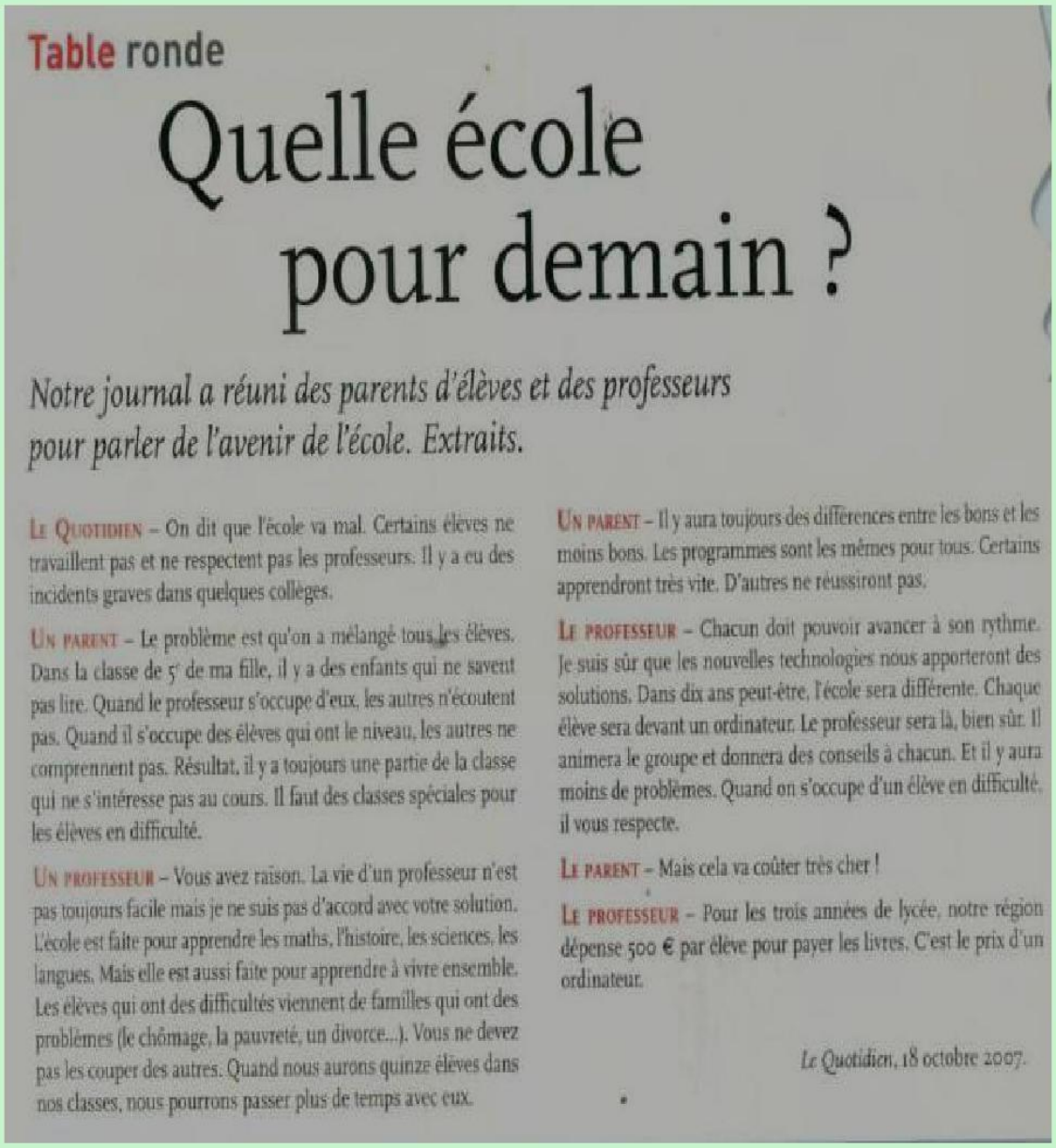

(Figura 1) 
Il s'agit d'un article sous-forme d'une table ronde (Fig.1) Nous n'avons pas d'information sur son auteur et nous n'avons pas trouvé cet article dans les archives du journal. Malgré cela, nous ne pouvons pas affirmer que ce document est fabriqué, car il a été publié dans le journal Le Quotidien, en 2007.

L'article est accompagné d'un titre "Quelle école pour demain ? », d'une phrase explicative «Notre journal a réuni des parents d'élèves... » et des mots soulignés en rouge et en gris « table ronde » en haut du document. Tous ces outils doivent être cités par l'enseignant, car ils favorisent la compréhension du texte et facilitent son exploitation.

L'article propose une discussion à propos de l'avenir de l'école où chaque participant donne son opinion sur plusieurs sujets, tels que le prix des études, la formation des professeurs, etc. Le manque de contextualisation est très évident et le lecteur ne peut pas repérer l'article dans le temps et dans l'espace : parle-t-on d'une école publique ou privée ? Peut-on affirmer que la réalité de l'école est la même dans toute la France?

L'article nous semble assez inesthétique. Sans image et sans couleur, il ne devient pas intéressant aux yeux des apprenants. Nous pensons que le manuel n'a fait aucun effort pour garder la forme originale du document : image, contexte. Malgré cela, le vocabulaire est accessible, le langage est correct et la présentation du texte est claire.

Écho a selectionné quelques extraits les plus intéressants de l'article et les a mis dans le manuel. Cette sélection est nécessaire et légitime car, il n'est pas possible de mettre un grand document dans une méthode de langue. En revanche, il devient capital d'informer au lecteur que le document a été modifié. Les mots « d'après », « extrait de », mis à la source, servent à indiquer que le document a été transformé.

La proposition des activités la plus intéressante est l'organisation d'une table ronde sur l'avenir de l'école dans le pays d'origine des apprenants. C'est une activité essentielle dans la mesure où elle favorise une communication réelle en classe. Les apprenants s'inspirent de l'article lu et échangent leurs opinions à propos d'un sujet commun présent dans leur pays. C'est un exercice qui vise à développer les compétences orale et interculturelle.

\section{L'interaction en classse produite par l' " article »}


Dans l'extrait ci-dessous, l'enseignante organise une table ronde au sein de classe. La table ronde axée sur « l'avenir de l'école au Brésil » était animée par l'enseignante (secrétaire). Celle-ci a distribué les rôles : A2 (professeur), A3 (journaliste), A4 (parent) et A5 (Ministre).

\section{Extrait : Table ronde sur le thème : Quelle école pour demain ?}

1 - E: ((secrétaire $))$ la secrétaire parle aussi + alors + là + vous dites que l'école ne doit pas former seulement les élèves mais aussi les parents + c'est encore une autre chose $+\mathrm{il} \mathrm{doit}+\mathrm{il}$ faut + je ne sais pas + qu'est-ce que tu veux dire/

2 - A3: ((journaliste)) il faut avoir une connexion avec l'école et les parents

3 - E: ((secrétaire $)<+$ il faut avoir une bonne connexion entre l'école et les parents

4 - A3: ((journaliste $)) \&$ il faut avoir une bonne connexion entre l'école et les parents pour une meilleure éducation des enfants

5 - A5: ((ministre)) mais + le problème c'est qu'il y a l'absence de l'argent pour investir + les problèmes sont les structures de l'école

6 - A3: ((journaliste)) nous payons

7 - E : ((secrétaire $))<+$ payons

8 - A3 : ((journaliste)) payons beaucoup d'argent pour les taxes du pays + si les salaires

9 - E: ((secrétaire $))$ allons simplifier les structures + les salaires doivent

10 - A3 ((journaliste)) les salaires doivent que ser meilleurs

11 -E ((secrétaire $))<+$ les salaires doivent être plus élevés

12 - A3: ((journaliste)) \& les professeurs travaillent meilleurs + plus motivés et éduquer des enfants + les professeurs sont beaucoup fatigués

13 - E: $(($ secrétaire $))<+$ très fatigués

14 - A3 : ((journaliste $))$ pour travailler beaucoup et plus de réponse éducative + plus des enfants + c'est ça + ils sont fatigués.

15 - A5: ((ministre $))$ mais + par la constitution + nous donnons $18 \%$ de budgets de l'union + ça c'est beaucoup d'argent + mais *geste de voler* $(($ rires $))$

L'enseignante est médiatrice de la discussion. Elle intervient en ajoutant des commentaires, en faisant des requêtes, en demandant des reformulations, etc. En premier lieu, nous soulignons l'interpellation : l'enseignante adresse la parole à la journaliste pour demander une reformulation de son commentaire (1). Puis, nous citons l'hétérocorrection: l'enseignante corrige la conjugaison du verbe payer (7), elle conseille le changement des termes «élevé» au lieu de «mieux» (11) et «très» au lieu de «beaucoup» (13). En dernier, nous mettons en exergue une action importante pour le déroulement de l'interaction : l'enseignante suggère une simplification de la structure de l'énoncé (9).

Dès le début du débat, on remarque l'engagement des élèves. Malgré leur vocabulaire restreint et leur niveau débutant (A.1.1), ils sont à l'aise avec la discussion. Cela ressemble à une conversation ordinaire, chacun essaie de défendre son point de vue. Il faut mettre en évidence le 
pouvoir de persuasion des étudiants. Les réponses sont toujours accompagnées de justifications «il faut avoir une bonne connexion entre l'école et les parents pour une meilleure éducation des enfants » (4) ou des critiques et marques d'ironie " mais + par la constitution + nous donnons $18 \%$ de budgets de l'union + ça c'est beaucoup d'argent + mais *geste de voler* ((rires))» (15).

$\mathrm{Au}$ cours de l'interaction, il y a eu une progression du thème. Chaque participant a cité les problèmes du système d'enseignement brésilien, la distance entre l'école et les parents, la rémunération des professeurs, la corruption dans le système scolaire qui affectent directement l'avenir de l'école, le rythme des heures de travail des professeurs, etc.

Certains chercheurs défendent l'idée selon laquelle les DAs sont « trop difficiles» pour les débutants. Nous réfutons cette idée, car comme on peut le voir dans l'extrait ci-dessus, le document authentique a permis non seulement le travail d'exposition (compréhension), de traitement (repérage) et de fixation (systématisation), mais aussi un travail de production (réemploi).

Nous sommes certains qu'il encourage la prise de parole chez les apprenants. Bien que ceux-ci n'aient pas assez de vocabulaire, ils se sentent concernés par la conversation et ils ont envie de s'exprimer malgré leur timidité. De plus, nous estimons que le DA est source d'inspiration pour des activités de production. Il permet que la classe de langue soit un lieu d'une véritable conversation réelle et authentique.

\section{$5^{\text {ème }}$ cours - Document Article}




\section{Environ 35000 concurrents au départ du Marathon de Paris}

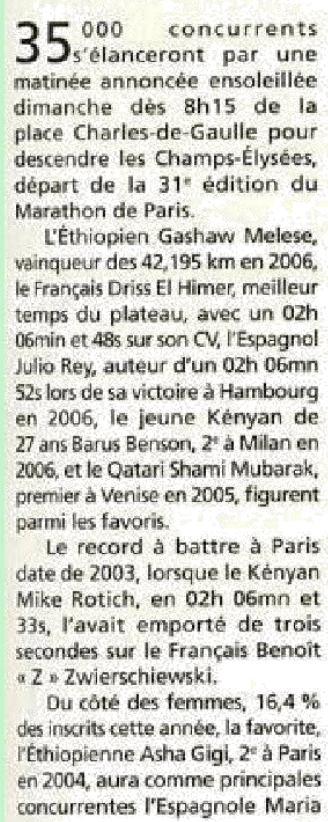

Abel, vainqueur a Francfort en 2002, ou une autre Ethopienne, Tafa Magarsd, vainqueur a Dubaicette annee

Apres les 36680 inscrits de: 2006, pour la $30^{\circ}$ edition, les organisateurs ont arrêté les dossards au numéro 35000 cette annee, et les Francais sont largement en téte parmi les 87 pays participants, avec $72 \%$ des inscrits. Le contingent étranger sera mené par quelque 3000 Britarniques et un peu plus de 1000 Italiens, la surprise venant de la presence de 459 Brésiliens.

Le parcours, entièrement trace sur la rive droite de la Seine, passe par la rue de Rivoli, la Bastille, le Bois de Vincennes, les quais rive droite, Ihippodrome d'Auteuil avant de remonter sur l'Acc de Triamphe par l'avenue Foch.

Les organisateurs ont prevu de distribuer pas moins de 17 tonnes de bananes, autant d'oranges, 2 tonnes de fruits secs, 6 tonnes de pommes et 436,800 bouteilles d'eau AfP (Agence France Pressel

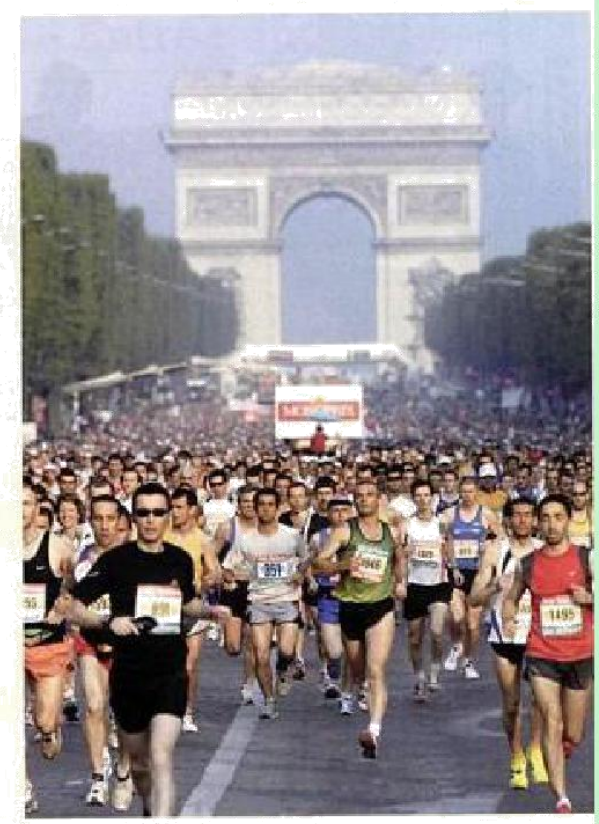

(Figura 2)

L'article (Fig.2) a été publié dans AFP en 2007. Cet article ne cite pas l'auteur. En revanche, la connaissance de celui-ci donne des indices sur le contenu ou du moins l'orientation du contenu du document. Le but de l'article est de délivrer l'information suivante « le Marathon de Paris aura lieu le dimanche dès 8 h15 ».

Il s'agit d'un article dans lequel le journaliste rend compte de l'événement. Son contenu répond aux questions : qui (concurrents), quoi (Marathon), où (Paris), quand (2007), comment (parcours adopté). L'article n'informe pas explicitement les raisons pour lequelles des milliers de personnes participent à un Marathon. Ainsi, c'est au lecteur de réfléchir sur les raisons qui poussent de nombreuses personnes à participer à cette course.

L'attaque est la première phrase de l'article. Elle doit surprendre le lecteur et l'inciter à lire la suite. Cette phrase donne l'essentiel de l'information « 35000 concurrents s'élanceront par une matinée...». Dans le corps du texte, chaque paragraphe contient un sous-thème : quels sont les candidats favoris, quel est le record à battre masculin et à battre féminin, quel est le pourcentage des participants Français et des étrangers, quel est le parcours à réaliser par les coureurs, quelle est la nourriture qui sera distribuée. L'auteur respecte la chronologie des faits et des propos et son but est de donner les informations brutes, sans analyse. 
L'article est convenablement présenté avec une mise en page esthétique, c'est-à-dire qu'il permet au lecteur d'accéder facilement à l'information. Cet article comporte : un titre que privilégie la nominalisation et il est informatif (ne cherche qu'à renseigner le lecteur); le corps, c'est-à-dire l'article en lui-même. Celui-ci suit un plan très précis et présente une séparation en parties; élément iconographique. Le vocabulaire et le style de la rédaction sont assez accessibles.

Nous estimons que cet article est approprié aux besoins linguistiques et culturels des apprenants du niveau A2. L'article a un vocabulaire accessible et si, parfois, il y a des mots obscurs, cela n'empêche pas l'élève de repérer les idées principales abordées. Nous avons questionné les étudiants de l'Alliance Française sur leur attitudes face à des articles de presse difficiles sur Internet et nous citons ici une opinion ; « je fais une compréhension globale de l'article parce que c'est une opportunité d'apprendre plus sur la langue comme des nouveaux mots ».En ce sens, nous partageons l'opinion de l'un des étudiants de l'Alliance Française qui affirme que l'intérêt de travailler avec les DAs est d' « apprendre avec la réalité, informellement $»$.

\section{L'interaction en classse produite par l' "article »}

L'extrait ci-dessous est l'introduction du cours consacré à la lecture et à la compréhension de l'article 35000 concurrents au départ... La mission première de l'enseignant consiste à susciter l'intérêt des élèves au sujet de l'article. Ainsi, l'enseignante propose une activité préalable et demande à A2 de raconter sa participation au Marathon de Paris en 2011. Son but est de mettre les étudiants en mouvement, leur permettre de prendre des repères, de construire du sens en se donnant une première représentation de la tâche. Voici un extrait du cours :

\section{Extrait : L'expérience de l'apprenant A2 au Marathon de Paris}

1- E : c'est quoi/ dis à tes camarades de classe + qu'est-ce que c'est/

2- A2 : cette photo est la Marathon de Paris

3- E : c'est le Marathon de Paris auquel

4- A2 : auquel j'ai participé en 2011

$5-\mathrm{E}:$ en 2011 + voilà

6- A2 : c'est pour ça que j'ai dit que j'ai dit que c'est merveilleuse $7-\mathrm{E}$ : voilà c'est merveilleux

8- A2 : que c'est merveilleux/ ahhhh + oui la Marathon 
9- $\mathrm{E}$ : voilà le Marathon $+\mathrm{A} 2+$ qu'est-ce que tu peux nous raconter + moi + j'ai jamais participé à un Marathon + vous avez déjà participé à un Marathon [au groupe] /

10- Gr : *geste de négation*

$11-\mathrm{E}$ : vous faites du jogging/ non/

12- Gr : *geste de négation*

13- E : Parle de cette expérience

14 - A2 : c'est une expérience merveilleuse

((rires)) 15- E : ((rires))

16- A2 : il y a beaucoup de gens + une energie très positive + tous les gens sont

heureuses $17-\mathrm{E}:<+$ heureux

18 - A2 : tout le monde est heureux + bien de santé

19 - E : en pleine forme + bien disposé

20 - A2 : en pleine forme + tous les gens sont belles

21 - E : sont beaux

22 - A2 : et le che $+>$ le parcours de la Marathon

23 - E : du Marathon

24 - A2 : est bel $+>$ est beaux + très beaux

25 - E : c'est Paris + n'est pas/ qu'est-ce que n'est pas bon à Paris /

26 - A2 : le chemin $+>+$ pendant le chemin + il y a beaucoup de monuments très importants de Paris + de

Paris + l'ambiance 27

- E : de l'ambiance

28 - A2 : de l'ambiance et le temps + la température est très

agréable 29 - E : c'est à quel époque de l'année/

30 - A2 : [abril] + elle a passé + elle a passé + semaine dernière

31 - E : ahhhh + c'était la semaine dernière à Paris/

32 - A2 : oui + il y a trois Marathons suivants importants

33 - E : 3 Marathons qui se succèdent

34 - A2 : La Marathon de Paris + la Marathon de Boston + malhereusement + vous avez vu qu'est-ce qui s'est passé [ les attentats le 15 avril 2013]

35 - E : c'est la catastrophe

36 - A2 : et la semaine suivante + la semaine prochaine + la Marathon de London

37 - E : le Marathon de Londres/

38 - Gr : (( murmuré) $)$

39 - E : qu'est-ce que passe/

40 - A6 : je ne savais pas que cette bombe a été

$41-\mathrm{E}:$ éclatée + éclatée + c'était à la fin + à la fin + à la rivière

42 - A2 : dans le kilomètre 40

43 - A4 : ils n'ont pas eu le temps de traverser la ligne de...

44 - E : d'arrivée

Tout d'abord, la stratégie de l'enseignante : valoriser les savoirs et la parole d'une étudiante. À titre d'exemple, nous citons la première réplique où l'enseignante invite A2 à parler du thème du cours : «c'est quoi ? Dis à tes camarades de classe. Qu'est-ce que c'est ?». Dans la troisième réplique, l'enseignante initie un énoncé et attend que A2 le complète avec son expérience « c'est le Marathon de Paris auquel.. ». La stratégie d'explorer une expérience 
personnelle d'une étudiante est une opportunité de donner aux apprenants des occasions de partager des idées, de préciser et de développer leurs idées personnelles et celles des autres.

$\mathrm{Au}$ cours de l'interaction, on constate que A2 se sent à l'aise pour partager son expérience « il y a beaucoup de gens + une energie très positive... (16) ; " pendant le chemin + il y a beaucoup de monuments très important de Paris... » (26). Ce partage de l'expérience vécue par A2 va, sans doute, promouvoir une discussion positive au sein du groupe. Les apprenants formulent des idées, écoutent attentivement ce que disent les autres, font preuve de sens critique et adoptent des points de vue ou des arguments pertinents.

Pour A2 prendre la parole c'est une opportunité de se remarquer en classe. Oser participer et se placer sous le regard d'autrui est une occasion de marquer son opinion dans l'interaction. Ainsi, A2 s'exprime d'un ton clair et il n'a pas peur de commetre des erreurs, par exemple «cette photo est la Marathon de Paris» (2) et « il y a trois Marathons suivants importants » (32) ; de son côté, l'enseignante la corrige soit à partir de reformulations « Marathons qui se succèdent $\gg(33)$.

Il faut souligner la fonction des DAs comme un instrument de travail qui permet l'insertion des apprenants dans une communication réelle. Dans la réplique 34, A2 énumère les Marathons du monde et cite les attentats du Marathon de Boston survenu en 2013. Ainsi, l'article favorise l'entrée des événements réels en cours. Les attentats du Marathon de Boston incitent les étudiants à exprimer leur individualité et de développer leurs idées personnelles : A6 s'etonne avec la tragédie « je ne savais pas que cette bombe a été éclatée » (40), A4 regrette l'interruption du Marathon « ils n'ont pas eu le temps de traverser la ligne d'arrivée » (43).

Cet article semble être efficace pour inviter les apprenants à communiquer en langue étrangère. Comme on vient de le voir, il a plusieurs avantages : c'est un outil de motivation dans la mesure où l'apprenant peut faire un lien entre le contenu véhiculé et ses expériences personnelles, apporte des aspects réels et actuels de la culture française et permet un travail basé sur la dimension linguistique, culturelle et pragmatique.

\section{Conclusion}

Cette étude nous a permis d'exploiter la notion de DAs : origine, variétés, avantages et insertion dans le cours de langue. À partir de données analysées, nous avons constaté que les DAs 
s'intègrent facilement dans l'enseignement communicatif et constituent pour l'enseignement du FLE un matériel privilégié: ils présentent aux apprenants la France, son quotidien, ses actualités et surtout le « vrai français », c'est-à-dire qu'ils leur permettent d'accéder au langage tel quel il est utilisé par les natifs. En plus, ils offrent une image authentique et riche du monde extérieur et contribuent ainsi à développer chez l'apprenant une attitude favorable à l'égard de la langue et de la culture étrangères.

Dans l'enseignement/apprentissage des langues, l'importance des DAs est soulignée, car ils ont été conçus dans un objectif communicatif: ils fournissent aux apprenants des situations de communication variées et ils leur permettent de questionner, de s'informer, d'exprimer leur point de vue, d'argumenter, etc. En analysant les interactions en classe, nous avons constaté que les DAs favorisent un cours interactif et dynamique, car les étudiants se sentent impliqués par ces supports et ils ont envie de partager leurs points de vue. Dans la confrontation des opinions, une conversation naturelle est privilegiée, très proche de ce qu'on appelle échange réel. Nous pouvons en déduire que les documents authentiques sont de vrais outils qui amènent l'apprenant à approfondir le fonctionnement de la langue apprise et à s'immerger dans la culture ciblée.

Nous avons pu tester diverses stratégies pédagogiques auprès des professeurs de l'Alliance Française en ayant recours principalement aux DAs présents dans le manuel Écho. Celles-ci ont su tirer profit de ces supports en salle de langue, notamment par leur facteur motivationnel et socioculturel. À titre d'exemple, nous soulignons la stratégie d'encourager l'implication des apprenants (éveiller leur curiosité) et de déclencher la production orale grâce à un fil conducteur puis les orienter vers la discussion sur un thème Quelle école pour demain? (1er cours). En ce sens, nous considérons que l'enseignant est celui qui va introduire le DA, qui va amener la réflexion sur le thème abordé et qui va favoriser le travail en autonomie chez les apprenants.

L'usage des DAs en classe n'a pas été « traumatisant », c'est-à-dire que les étudiants ne se sont pas trouvés contraints par la présence d'autres collègues. Au contraire, face à un DA, ils se sentent à l'aise et n'ont pas une timidité excessive qui empêche la participation au cours. Il faut souligner que les DAs tiennent compte de l'environnement des apprenants. Autrement dit, ces mines d'or leur permettent de parler de leur monde, de leur culture, de leur point de vue, de leurs expériences individuelles, etc. Par conséquent, les étudiants se sent concernés dans 
l'interaction, acquièrent une méthodologie et développent un niveau minimum dans la compétence de prise de parole.

Il faut mettre en évidence qu'il y a une plus forte proportion de DAs que de documents fabriqués dans le manuel Écho. Cela renforce l'idée qu'il ne faut pas limiter le nombre de DAs dans les manuels destinés aux niveaux débutants : ils ont besoin aussi du contact avec de l'authentique ou du semi-authentique dans leur apprentissage. Selon l'une des enseignantes de l'Alliance Française, les DAs ont une place importante dans Écho «Il y en a dans chaque leçon. Dans la plupart des activités, elle essaie d'exploiter les DAs de manière la plus naturelle possible : c'est comme si l'apprenant lisait un article de journal... »

Nous avons souligné au début de notre étude, les avantages de l'utilisation de DAs: motiver les étudiants, travailler en parallèle l'apport linguistique et culturel, permettre une communication plus authentique. Ces avantages ont été confirmés dans les questionnaires remplis par les professeurs et par les étudiants. Ils ont souligné que les DAs ont plusieurs fonctions dans la classe : ils permettent d'aborder un thème, prolonger un cours à l'aide d'activités diverses et d'ouvrir la classe sur le monde extérieur. L'une des enseignantes de l'Alliance Française nous a affirmé l'intérêt de travailler avec cet outil «Quand on utilise le DA, on est dans un contexte plus proche du réel, où l'étudiant peut agir comme un vrai acteur».

Nous espérons avoir convaincu l'enseignant de FLE de se risquer à introduire les DAs dans son arsenal pédagogique. En effet, ils permettent à la fois de travailler la dimension culturelle et interculturelle, l'apport linguistique et le travail de plusieurs compétences, dont l'oral. Nous sommes convaincus que ces documents sont à la fois des outils qui aident à l'apprentissage de la langue et de la culture française, une clef qui rend le cours plus vivant et qui suscite la prise de parole au sein de la classe et une source de diversité qui enrichit le manuel de FLE.

\section{Références}

BERARD, E. L'approche communicative - Théorie et pratiques. Paris: Cle International, 1991.

BESSE, H. Polémique en didactique. Paris: Cle International, 1980.

CHARAUDEAU, P. Le contrat de communication dans la situation classe. Inter-Actions Halté, 1993. Disponível em: <http://www.patrick-charaudeau.com/Le-contrat-de-communicationdans.html > Acesso em: 15 août. 2013. 
CICUREL, F. \& Bigot, V. Les interactions en classe de langue. Le Français dans le monde. Numéro spécial Recherches et Applications, p. 42-53, 2005.

CICUREL, F. La classe de langue un likeu ordinaire, une interaction complexe. Acquisition et interaction en langue étrangère, 2002. Disponível em : <http://aile.revues.org/801> Acesso em: 20 mai. 2013.

International, 1985.

Parole sur parole ou le métalangage dans la classe de langue. Paris : Cle

CONSEIL DE L'EUROPE. Cadre européen commun de référence pour les langues. 2. éd. Paris : Didier, 2001.

COURTILLON, J. Élaborer un cours de FLE. Paris: Hachette, 2003.

CUQ, J.-P. ; Gruca, I. Cours de didactique du français langue étrangère et seconde. Grenoble: PUG, 2005.

CUQ, J.-P. Dictionnaire de didactique du français langue étrangère et seconde. Paris: Cle International, 2003.

DABÈNE, Louis et al. Variations et rituels en classe de langue. Paris: Hatier-Credif, 1990.

GOFFMAN, E. La mise en scène de la vie quotidienne. Paris: Minuit, 1973.

KERBRAT-ORECCHIONI, C. Les interactions verbales, Approche interactionnelle et structure des conversations. Paris : Armand Colin, 1998.

LOI $n^{\circ}$ 57-298, de 11/03/1957. La loi sur la propriété littéraire et artistique. Disponível em: <http://admi.net/jo/loi57-298.html > Acesso em: 10 mars 2012.

MOIRAND, S. Enseigner à communiquer en langue étrangère. Paris: Hatier, 1982.

VION, R. La communication verbale, analyse des interactions. Paris : Hachette, 1992.

ZARATE, G. Représentations de l'étranger et didactique des langues. Paris: Didier, 1994. Enseigner une culture étrangère. Paris : Hachette, 1996.

Recebido: 014.06.2015

Aceito: 10.08 .2015 